\title{
Beta-2-microglobulin expression in the liver after liver transplantation
}

\author{
S G HUBSCHER, * D H ADAMS, E ELIAS
}

From the *Department of Pathology, University of Birmingham, and the Liver Unit, Queen Elizabeth Hospital, Birmingham

SUMMARY The distribution of major histocompatibility complex (MHC) class 1 antigens was studied in the liver after transplantation by immunoperoxidase staining for $\beta$-2-microglobulin $\left(\beta_{2} \mathrm{~m}\right)$, a subunit of the class 1 antigen system. Paraffin wax sections were examined from 25 "time zero" biopsy specimens, taken immediately after insertion of the graft, and 87 biopsy specimens taken after transplantation in seven diagnostic categories: acute cellular rejection $(n=22)$; resolving acute rejection $(n=8)$; chronic rejection $(n=22)$; pure cholestasis $(n=14)$; ischaemia/infarction $(n=5)$; biliary obstruction $(n=8)$; massive haemorrhagic necrosis $(n=8)$. Staining was graded semiquantitatively on a scale of $0-3+$ in bile ducts, hepatocytes, sinusoidal lining cells and vascular endothelium. Using the "time zero" biopsy specimens as a baseline for comparison, increased expression of $\beta_{2} \mathrm{~m}$ was seen in bile ducts, hepatocytes, and endothelial cells after transplantation. These changes were most pronounced in cases of rejection but also occurred in other graft conditions. The degree of hepatocyte and endothelial staining was significantly higher in cases of rejection and massive haemorrhagic necrosis than in the other categories.

These findings may have implications for the pathogenesis and diagnosis of rejection of the transplanted liver.

Although there is increasing agreement over the histological features that characterise liver allograft rejection, ${ }^{1-6}$ the underlying mechanisms remain poorly understood. Previous studies have shown changed expression of class I major histocompatibility complex (MHC) antigens in the liver after transplantation. ${ }^{7-9}$ It is not clear, however, whether this is a specific feature of rejection or a non-specific finding seen in other circumstances following transplantation.

Retrospective analysis of biopsy material obtained during the first five years of transplantation procedures in Birmingham highlighted several "posttransplant" syndromes, each with distinctive clinical and pathological features. ${ }^{10}$ This study aimed at examining the distribution of class I antigens in biopsy specimens from each of these various syndromes and at seeing whether a specific pattern of expression was present in cases of rejection.

To assess the distribution of class I antigens we investigated the pattern of $\beta_{2}$-microglobulin $\left(\beta_{2} \mathrm{~m}\right)$ staining in paraffin wax embedded biopsy material using an immunoperoxidase method. $\beta_{2} \mathrm{~m}$ is a low molecular weight protein which has been identified as the light chain of HLA A, B, and C antigens. It is non- covalently attached to MHC class I heavy chains and is essential to their serological specificity and cell surface expression. ${ }^{11}{ }^{12}$ Unlike other components of the class I antigen system, $\beta_{2} \mathrm{~m}$ can be readily shown in paraffin wax embedded tissue sections.

\section{Material and methods}

CLINICAL AND HISTOLOGICAL DATA

One hundred and twelve specimens were examined from 32 transplantations carried out on 28 patients. These included 25 "time zero" biopsy specimens obtained immediately after insertion of the graft and 87 specimens taken between five and 876 days after transplantation. Transplant material comprised 78 percutaneous needle biopsy specimens, four wedge biopsy specimens taken at repeat laparotomy, and five surgically removed livers obtained at retransplantation. All tissues were fixed in formalin and processed routinely into paraffin wax blocks. Biopsy specimens were sectioned at four levels and stained with haemotoxylin and eosin, together with other special stains as appropriate.

All of the "time zero" biopsy specimens showed minor histological abnormalities. These included sur- 
gical hepatitis $(n=23)$, hepatocyte degeneration (cloudy swelling, fine cytoplasmic vacuolation, macrovesicular fat droplets; $n=21$ ), hepatocyte necrosis (focal/confluent; $\mathrm{n}=11$ ), cholestasis $(n=11)$. These changes were generally confined to perivenular areas and peripheral acinar zones. Inflammation related to surgical trauma was more widespread.

Biopsy specimens taken from transplanted livers were designated one of seven diagnostic categories on the basis of characteristic histological features supported by compatible clinical, biochemical, and radiological findings.

Acute rejection (16 cases, 22 biopsy specimens, days 5-44). This was characterised by a combination of (i) portal inflammation, (ii) bile duct damage, and (iii) venous endothelial inflammation.

Resolving acute rejection (six cases, eight biopsy specimens, days 12-62). Histological features of acute rejection resolved in these cases after treatment with high dose steroids and were accompanied by clinical and biochemical signs of recovery.

Chronic rejection (five cases, 22 biopsy specimens, days 10-164). This was characterised by recurrent episodes of portal inflammation, mainly mononuclear, with loss of bile ducts. Four cases progressed to end stage liver disease; two were retransplanted, the other two died. Both transplant hepatectomy specimens showed widespread foam cell arteriopathy as previously described in cases of chronic rejection. ${ }^{113}$ The fifth patient was alive at the time of writing with stable graft function 15 months after the original transplantation. Pure cholestasis (four cases, 14 biopsy specimens, days 6-201). Severe cholestasis with no other histological abnormalities was seen in these cases. One patient was subsequently found to have sepsis as the likely cause for cholestasis. The cause of cholestasis in the other three was not known.

Ischaemia/infarction (five cases, four biopsy specimens, one hepatectomy, days 5-30). These cases all showed histological features of ischaemic necrosis. ${ }^{14}$ This was due to hypoperfusion or hypotension in three patients and hepatic artery thrombosis in the other two.

Biliary obstruction (four cases, eight biopsy specimens, days 45-876). These all had radiological or surgical evidence of bile duct obstruction with compatible histological changes.

Massive haemorrhagic necrosis (six cases, four biopsy specimens, four transplant hepatectomies, days 5-24). These cases were characterised clinically by fulminant graft failure of sudden onset and uncertain aetiology. The main histological findings were those of massive haemorrhage and hepatocyte necrosis with only a sparse cellular infiltrate. The cause of this lesion was not apparent histologically but there were no features to suggest an ischaemic or infective aetiology. ${ }^{15}$

In each of the biopsy specimens included in this study a single cause or pattern of graft dysfunction was evident. Several cases, however, had biopsy specimens which fell into different diagnostic categories at different times - for example, many cases with early biopsy specimens showing features of acute rejection had subsequent biopsy specimens which showed other patterns of graft damage.

\section{IMMUNOHISTOLOGICAL METHODS}

Staining for $\beta_{2} \mathrm{~m}$ was carried out using a three stage peroxidase-antiperoxidase (PAP) technique. Sections were dewaxed and trypsinised $(0.1 \mathrm{~g}$ trypsin in $100 \mathrm{ml}$ buffer) at $37^{\circ} \mathrm{C}$ for 30 minutes. These were then incubated with polyclonal rabbit anti-human $\beta_{2} \mathrm{~m}$ ( 1 in 30 for one hour), swine anti-rabbit immunoglobulin (1 in 80 for 45 minutes), and rabbit-PAP ( 1 in 80 for 40 minutes). All antibodies and trypsin were diluted in phosphate buffered saline (PBS). The final reaction product was visualised with $3^{\prime}-5^{\prime}$ diaminobenzidine and hydrogen peroxide. All antisera used were obtained from Dakopatts, Denmark. Negative controls were obtained by substitution of the primary antiserum with PBS and by absorption with $\beta_{2} \mathrm{~m}$ antigen (Sigma).

Examination of stained sections was carried out simultaneously by two observers (SGH and DHA). Staining was specifically assessed in the following four structures: bile ducts; hepatocytes (membranous staining); sinusoidal lining cells; and endothelial cells. It was often impossible to distinguish Kupffer cells from other sinusoidal lining cells, and endothelial staining in this study refers specifically to the endothelium of hepatic arteries, portal venules, and hepatic venules. The degree of positive staining was further graded semiquantitatively as follows: $\mathrm{O}=$ negative, $+/-=$ minimal/only occasional cells positive; $+=$ weak/ focal; $++=$ moderate/patchy in distribution; $+++=$ intense/generalised. In the case of membranous hepatocyte staining the extent of positive staining was graded thus: $+=<10 \%$ of hepatocytes positive; $++=10-50 \%$ positive; $++t=>50 \%$ positive. Finally, a "mean staining score" was calculated for each histological category by scoring points for each of the staining grades described above $\left(0=0\right.$ points, $+/-=\frac{1}{2}$ point, $+=1$ point, $++=2$ points, $+++=3$ points) and dividing the total by the number of biopsy specimens containing the structure to be assessed. If a particular feature could not be assessed in one or more specimens (such as bile ducts in cases of chronic rejection, or hepatocytes in cases with extensive infarction) the total score was divided by the number of remaining specimens in which the given feature could still be assessed. The staining scores in different histological categories were compared statis- 
Table $1 \quad \beta_{2} m$ staining in bile ducts

\begin{tabular}{|c|c|c|c|c|c|c|c|}
\hline \multirow{2}{*}{$\begin{array}{l}\text { Histological } \\
\text { diagnosis }\end{array}$} & \multirow{2}{*}{$\begin{array}{l}\text { No of } \\
\text { specimens }\end{array}$} & \multicolumn{5}{|c|}{ Degree of staining } & \multirow{2}{*}{$\begin{array}{l}\text { Mean } \\
\text { staining score }\end{array}$} \\
\hline & & $O$ & $+1-$ & + & ++ & +++ & \\
\hline $\begin{array}{l}\text { Time zero } \\
\text { Acute rejection } \\
\text { Resolving acute rejection } \\
\text { Chronic rejection } \\
\text { Pure cholestasis } \\
\text { Ischaemia/infarction } \\
\text { Biliary obstruction } \\
\text { Massive haemorrhagic necrosis }\end{array}$ & $\begin{array}{c}25 \\
22 \\
8 \\
16^{*} \\
14 \\
5 \\
8 \\
8\end{array}$ & $\begin{array}{l}2 \\
0 \\
0 \\
0^{*} \\
0 \\
0 \\
0 \\
0\end{array}$ & $\begin{array}{r}16 \\
1 \\
0 \\
0 \\
2 \\
0 \\
0 \\
0\end{array}$ & $\begin{array}{l}7 \\
5 \\
4 \\
5 \\
6 \\
3 \\
1 \\
1\end{array}$ & $\begin{array}{r}0 \\
10 \\
4 \\
10 \\
5 \\
2 \\
5 \\
5\end{array}$ & $\begin{array}{l}0 \\
6 \\
0 \\
1 \\
1 \\
0 \\
2 \\
2\end{array}$ & $\begin{array}{l}0.60 \\
1.98 \\
1.50 \\
1.75 \\
1.43 \\
1.40 \\
2.12 \\
2.12\end{array}$ \\
\hline
\end{tabular}

*Six biopsy specimens had no residual bile ducts

Table $2 \beta_{2}$ m staining in hepatocytes (membranous staining)

\begin{tabular}{|c|c|c|c|c|c|c|c|}
\hline \multirow{2}{*}{$\begin{array}{l}\text { Histological } \\
\text { diagnosis }\end{array}$} & \multirow{2}{*}{$\begin{array}{l}\text { No of } \\
\text { specimens }\end{array}$} & \multicolumn{5}{|c|}{ Degree of staining } & \multirow{2}{*}{$\begin{array}{l}\text { Mean } \\
\text { staining score }\end{array}$} \\
\hline & & $O$ & $+1-$ & + & ++ & ++ & \\
\hline $\begin{array}{l}\text { Time zero } \\
\text { Acute rejection } \\
\text { Resolving acute rejection } \\
\text { Chronic rejection } \\
\text { Pure cholestasis } \\
\text { Ischaemia/infarction } \\
\text { Biliary obstruction } \\
\text { Massive haemorrhagic necrosis }\end{array}$ & $\begin{array}{r}25 \\
22 \\
8 \\
22 \\
14 \\
3^{*} \\
8 \\
8\end{array}$ & $\begin{array}{l}17 \\
0 \\
0 \\
0 \\
0 \\
0^{*} \\
0 \\
0\end{array}$ & $\begin{array}{l}4 \\
0 \\
2 \\
0 \\
0 \\
0 \\
1 \\
0\end{array}$ & $\begin{array}{l}4 \\
7 \\
3 \\
5 \\
6 \\
3 \\
7 \\
1\end{array}$ & $\begin{array}{r}0 \\
12 \\
3 \\
10 \\
8 \\
0 \\
0 \\
0 \\
2\end{array}$ & $\begin{array}{l}0 \\
3 \\
0 \\
7 \\
0 \\
0 \\
0 \\
5\end{array}$ & $\begin{array}{l}0.24 \\
1.82 \\
1.25 \\
2.09 \\
1.57 \\
1.00 \\
0.94 \\
2.50\end{array}$ \\
\hline
\end{tabular}

*Two biopsy specimens had no viable hepatocytes

Table $3 \quad \beta_{2} m$ staining in endothelium

\begin{tabular}{|c|c|c|c|c|c|c|c|}
\hline \multirow{2}{*}{$\begin{array}{l}\text { Histological } \\
\text { diagnosis }\end{array}$} & \multirow{2}{*}{$\begin{array}{l}\text { No of } \\
\text { specimens }\end{array}$} & \multicolumn{5}{|c|}{ Degree of staining } & \multirow{2}{*}{$\begin{array}{l}\text { Mean } \\
\text { staining score }\end{array}$} \\
\hline & & $o$ & $+1-$ & + & ++ & +++ & \\
\hline $\begin{array}{l}\text { Time zero } \\
\text { Acute rejection } \\
\text { Resolving acute rejection } \\
\text { Chronic rejection } \\
\text { Pure cholestasis } \\
\text { Ischaemia/infarction } \\
\text { Biliary obstruction } \\
\text { Massive haemorrhagic necrosis }\end{array}$ & $\begin{array}{c}25 \\
22 \\
7 * \\
22 \\
14 \\
5 \\
8 \\
8\end{array}$ & $\begin{array}{l}0 \\
0 \\
1 \\
0 \\
0 \\
0 \\
0 \\
0\end{array}$ & $\begin{array}{l}9 \\
0 \\
1 \\
0 \\
1 \\
2 \\
3 \\
0\end{array}$ & $\begin{array}{r}14 \\
6 \\
5 \\
8 \\
8 \\
3 \\
4 \\
1\end{array}$ & $\begin{array}{r}2 \\
14 \\
0 \\
9 \\
5 \\
0 \\
1 \\
7\end{array}$ & $\begin{array}{l}0 \\
2 \\
0 \\
5 \\
0 \\
0 \\
0 \\
0\end{array}$ & $\begin{array}{l}0.90 \\
1.82 \\
0 \cdot 79 \\
1.86 \\
1.32 \\
0.80 \\
0.94 \\
1.87\end{array}$ \\
\hline
\end{tabular}

*ne biopsy specimen had no blood vessels

Table $4 \quad \beta_{2} m$ staining in sinusoidal lining cells

\begin{tabular}{|c|c|c|c|c|c|c|c|}
\hline \multirow{2}{*}{$\begin{array}{l}\text { Histological } \\
\text { diagnosis }\end{array}$} & \multirow{2}{*}{$\begin{array}{l}\text { No of } \\
\text { specimens }\end{array}$} & \multicolumn{5}{|c|}{ Degree of staining } & \multirow{2}{*}{$\begin{array}{l}\text { Mean } \\
\text { staining score }\end{array}$} \\
\hline & & $O$ & $+1-$ & + & ++ & +++ & \\
\hline $\begin{array}{l}\text { Time zero } \\
\text { Acute rejection } \\
\text { Resolving acute rejection } \\
\text { Chronic rejection } \\
\text { Pure cholestasis } \\
\text { Ischaemia/infarction } \\
\text { Biliary obstruction } \\
\text { Massive haemorrhagic necrosis }\end{array}$ & $\begin{array}{r}25 \\
22 \\
8 \\
22 \\
14 \\
5 \\
8 \\
7\end{array}$ & $\begin{array}{l}\mathbf{0} \\
\mathbf{0} \\
\mathbf{0} \\
\mathbf{0} \\
\mathbf{0} \\
\mathbf{0} \\
\mathbf{0} \\
\mathbf{0}\end{array}$ & $\begin{array}{l}2 \\
1 \\
0 \\
0 \\
0 \\
1 \\
0 \\
0\end{array}$ & $\begin{array}{l}7 \\
3 \\
1 \\
3 \\
5 \\
2 \\
1 \\
2\end{array}$ & $\begin{array}{r}15 \\
18 \\
7 \\
15 \\
9 \\
2 \\
5 \\
5\end{array}$ & $\begin{array}{l}1 \\
0 \\
0 \\
4 \\
0 \\
0 \\
2 \\
0\end{array}$ & $\begin{array}{l}1.64 \\
1.80 \\
1.87 \\
2.05 \\
1.64 \\
1.30 \\
2 \cdot 12 \\
1 \cdot 71\end{array}$ \\
\hline
\end{tabular}

*Not possible to assess properly in one biopsy specimen 


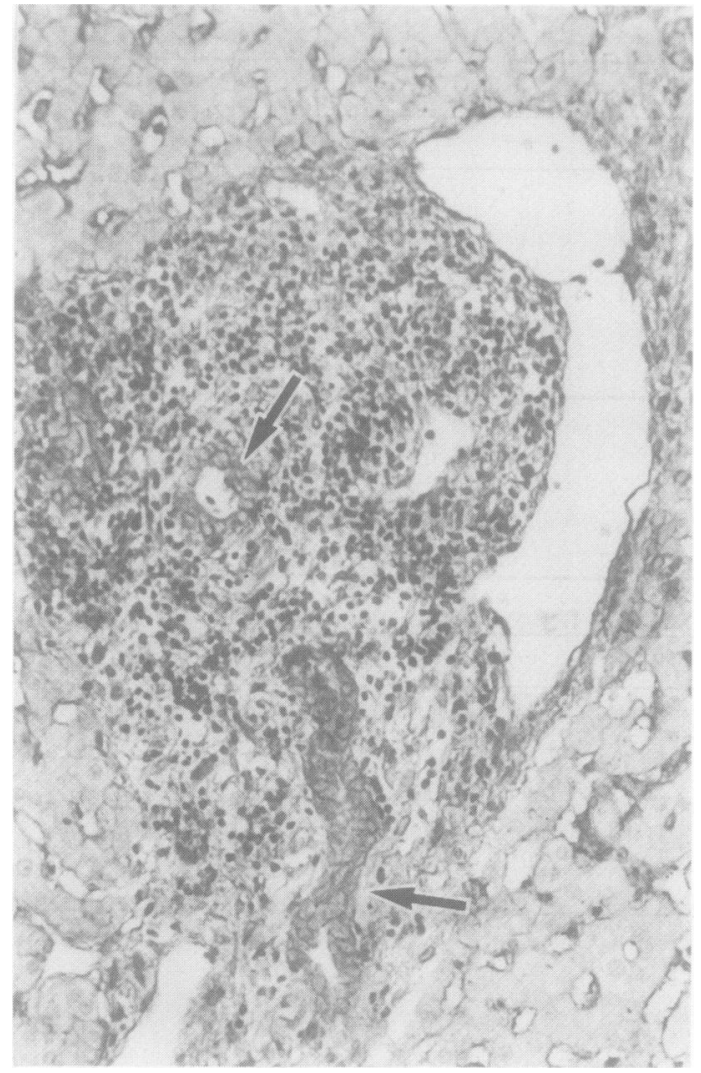

Fig 1 Bile duct $\beta_{2} m$ expression in acute rejection: (day 8 biopsy specimen) bile ducts (arrowed) show strongly positive membranous and cytoplasmic staining for $\beta_{2} m$. Diffuse staining is also present in hepatic sinusoids.

tically using the Mann-Whitney U test.

\section{Results}

Tables 1-4 summarise the staining pattern for $\beta_{2} \mathrm{~m}$ in each of the four structures that were specifically assessed. Positive immunoperoxidase staining was completely abolished by replacing the first antiserum with PBS and by absorption with $\beta_{2} \mathrm{~m}$ antigen.

\section{"TIME-ZERO" BIOPSY SPECIMENS}

A moderate degree of staining was present in sinusoidal lining cells. Weaker staining was seen in endothelial cells and bile ducts. Hepatocyte staining was absent in $70 \%$ of cases. The remaining $30 \%$ of biopsy specimens showed faint focal membranous staining, usually around central venules. The presence of membranous hepatocyte staining correlated with the presence and severity of cholestasis and hepatocyte necrosis in sections stained with haematoxylin and eosin.

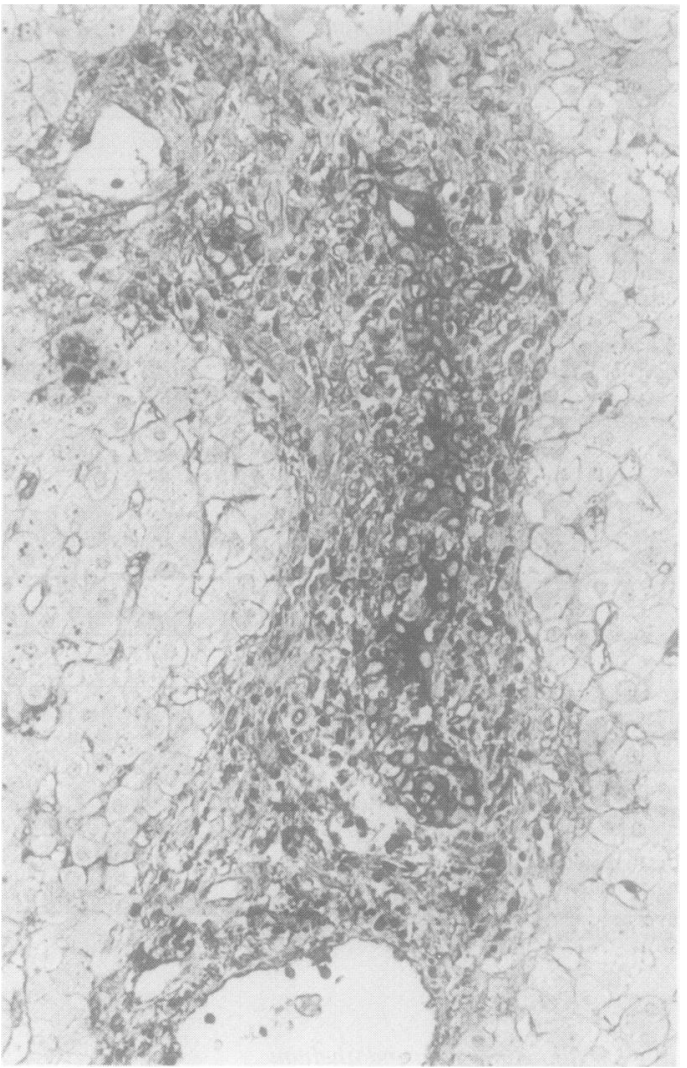

Fig 2 Bile duct $\beta_{2} m$ expression in early chronic rejection: (day 12 biopsy specimen). This shows intense biliary epithelial staining for $\beta_{2} m$. Bile ducts are effaced by inflammatory cells and were difficult to identify on sections stained by haemotoxylin and eosin.

\section{BIOPSY SPECIMENS AFTER TRANSPLANTATIONS \\ Bile ducts}

Bile duct expression of $\beta_{2} \mathrm{~m}$ was significantly increased in all transplant histological groups in comparison with the time-zero biopsy specimens ( $p<0.001$ for each category). Biliary epithelial staining was strongest on the basolateral membrane but was also present throughout the cytoplasm of bile ducts in most cases (figs 1 and 2). Staining of bile ducts in acute rejection was associated with positive staining of lymphoid cells in portal inflammatory infiltrates. Although increased bile duct expression of $\beta_{2} \mathrm{~m}$ was a feature of acute and chronic rejection (figs 1 and 2), it often persisted after portal inflammation had resolved and also occurred in non-rejection biopsy specimens (fig 3). Overall, there was no significant difference in the degree of staining among cases of rejection (acute or chronic) and nonrejection biopsy specimens (pure cholestasis, ischaemia, biliary obstruction). The degree of bile duct $\beta_{2} \mathrm{~m}$ expression was slightly higher in massive 


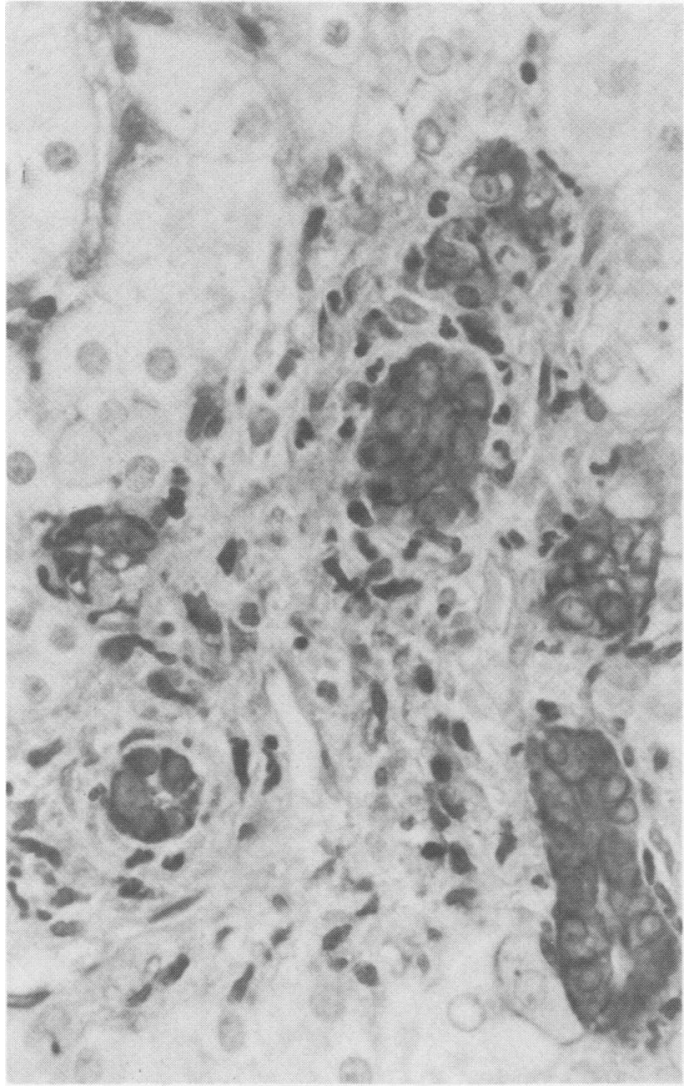

Fig 3 Bile duct $\beta_{2} m$ expression in pure cholestasis: (day 19 biopsy specimen). Portal inflammation has resolved following a previous episode of acute rejection. Bile ducts continue to display $\beta_{2} m$ antigens strongly. Conventional histological examination showed persistent perivenular cholestasis.

haemorrhagic necrosis than in non-rejection biopsy specimens $(\mathrm{p}<0.05)$.

\section{Hepatocytes}

Membranous expression of $\beta_{2} \mathrm{~m}$ in hepatocytes was significantly increased in all post-transplant groups in comparison with time-zero biopsy specimens ( $\mathrm{p}<$ 0.0001 for all categories except ischaemia-p $<$ $0.005)$. Hepatocyte staining was most prominent in perivenular areas but occasional periportal hepatocytes also stained positively (fig 4). It was commonly associated with cholestasis, many bile plugs also staining positively for $\beta_{2} \mathrm{~m}$ (fig 5). The overall degree of hepatocyte staining was higher in cases of rejection than in non-rejection biopsy specimens when these were considered as a whole $(\mathrm{p}<$ 0.005 for acute rejection, $\mathrm{p}<0.0005$ for chronic rejection). There was still an overlap between rejection and other post-transplant syndromes, however, when

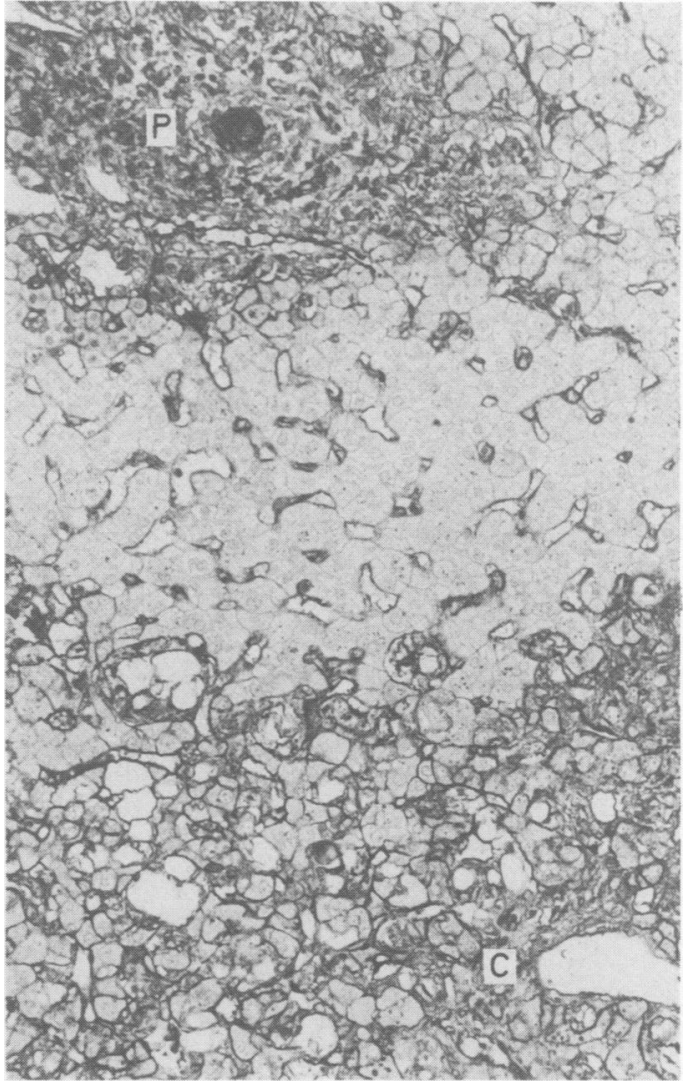

Fig 4 Membranous hepatocyte $\beta_{2} m$ expression: (day 12 biopsy specimen). Positive membranous staining for $\beta_{2} m$ is seen in numerous perivenular hepatocytes and a few periportal hepatocytes. (From a case of early chronic rejection) $(P=$ portal tract, $c=$ central vein $)$.

individual categories (such as pure cholestasis) were considered separately. Hepatocyte expression was also greater in cases of massive haemorrhagic necrosis than in non-rejection biopsy specimens $(\mathrm{p}<0.0005)$. An unusual and striking pattern of membranous hepatocyte staining was seen in two of the six cases of massive haemorrhagic necrosis. In both of these there was panacinar membranous staining entailing almost $100 \%$ of hepatocytes (fig 6). This degree of staining was not seen in any of the other post-transplant biopsy specimens.

\section{Endothelium}

Endothelial staining for $\beta_{2} \mathrm{~m}$ was significantly increased in cases of acute rejection $(p<0.00005)$, chronic rejection $(p<0.00001)$, pure cholestasis $(p<$ 0.01 ), and massive haemorrhagic necrosis ( $p<$ $0.0001)$ compared with time-zero biopsy specimens. Endothelial staining was not significantly affected in 


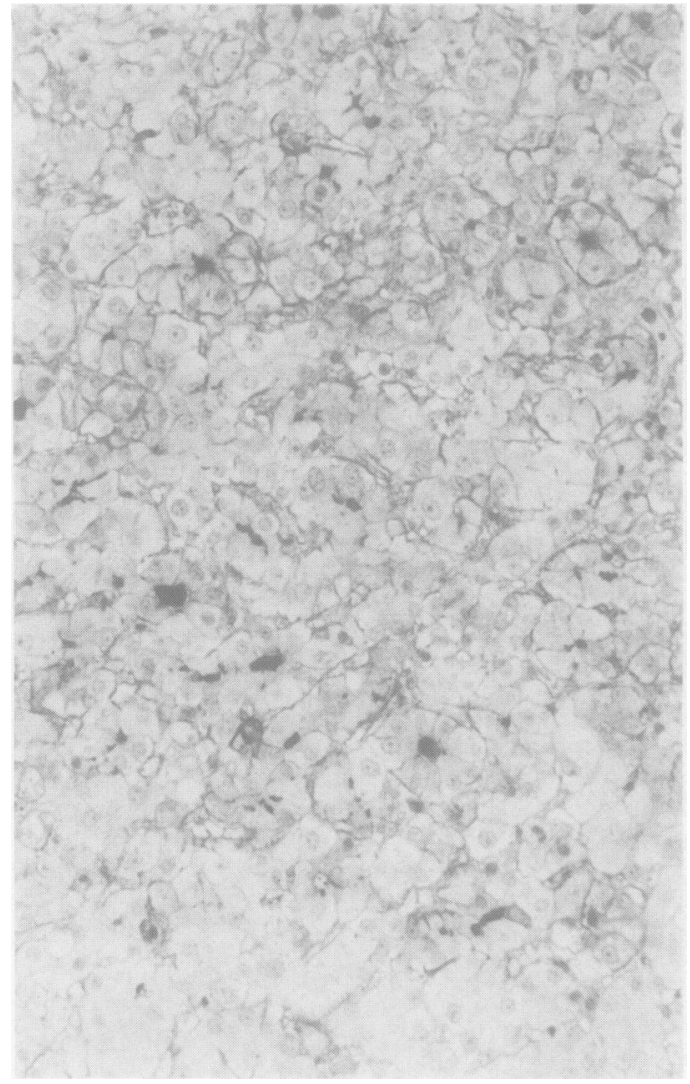

Fig 5 Membranous hepatocyte $\beta_{2}$ m expression associated with cholestasis: (day 13 biopsy specimen). This case of resolving acute rejection shows membranous hepatocyte staining associated with bile plugs, many of which also stain positively for $\beta_{2} m$.

the other three transplant categories (resolving acute rejection, ischaemia, biliary obstruction). Increased $\beta_{2} \mathrm{~m}$ staining in endothelium was mainly seen in portal and hepatic venules and was much less evident in hepatic arterioles. In cases of acute rejection $\beta_{2} \mathrm{~m}$ expression in venular endothelium was associated with infiltration by lymphoid cells, many of which also showed strongly positive staining for $\beta_{2} \mathrm{~m}$ (fig 7). Endothelial expression of $\beta_{2} \mathrm{~m}$ was significantly greater in cases of acute rejection ( $p<0.0001)$, chronic rejection $(\mathrm{p}<0.0005)$, and massive haemorrhagic necrosis $(\mathrm{p}<0.001)$ than in non-rejection biopsy specimens. These differences remained significant $(\mathrm{p}$ $<0.02$ ) when each of the non-rejection groups was considered separately.

Sinusoidal lining cells A slightly increased expression of $\beta_{2} \mathrm{~m}$ was observed in

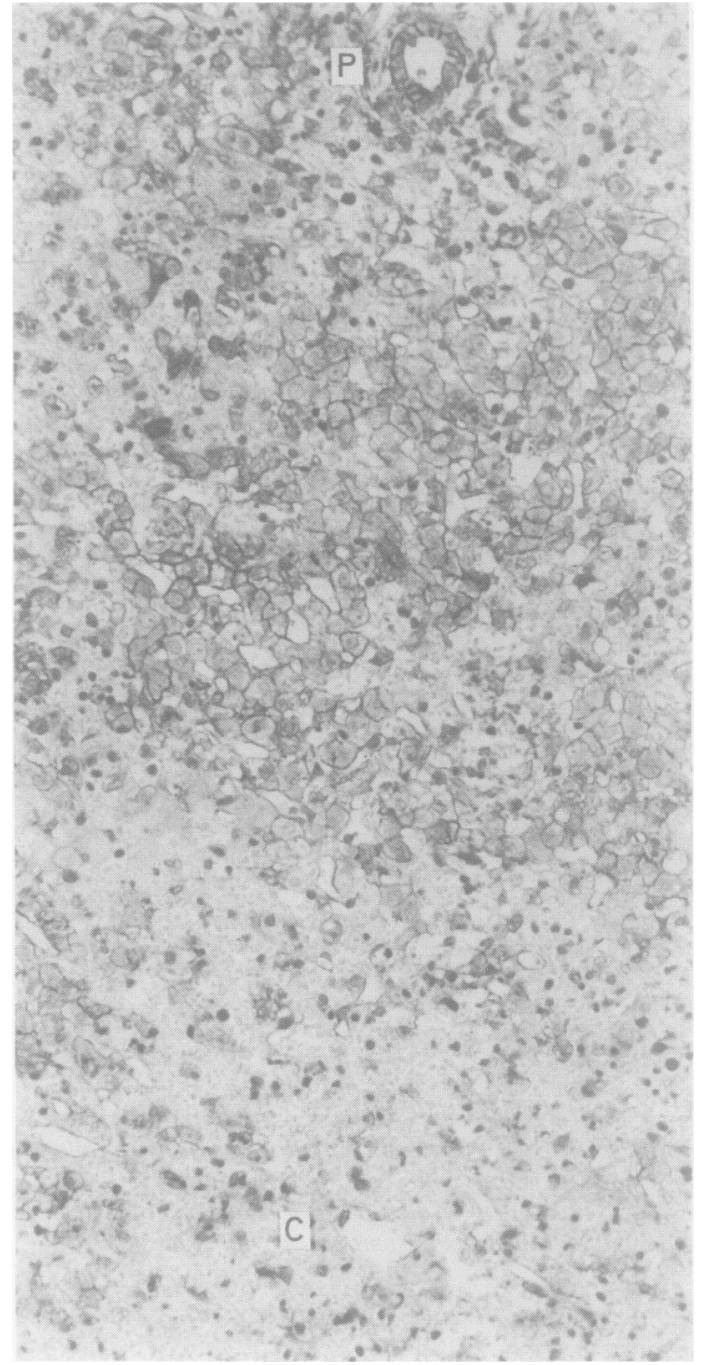

Fig 6 Panacinar hepatocyte $\beta_{2}$ m expression in massive haemorrhagic necrosis: (day 5 biopsy specimen). This biopsy shows an early lesion of massive haemorrhagic necrosis with perivenular congestion and early disruption of liver cell plates. There is striking panacinar membranous hepatocyte $\beta_{2} m$ display. Nearly all hepatocytes stain positively for $\beta_{2} m$. $(P=$ portal tract, $C=$ central vein).

cases of chronic rejection $(p<0.05)$ and biliary obstruction $(p<0.05)$ when compared with time-zero biopsy specimens. No significant increase was seen in the other post-transplant groups. $\beta_{2}$ m expression was also increased in cases of acute rejection compared with ischaemia ( $p<0.05)$, and in cases of chronic rejection compared with pure cholestasis $(p<0.05)$ and ischaemia $(p<0.05)$. No significant differences 


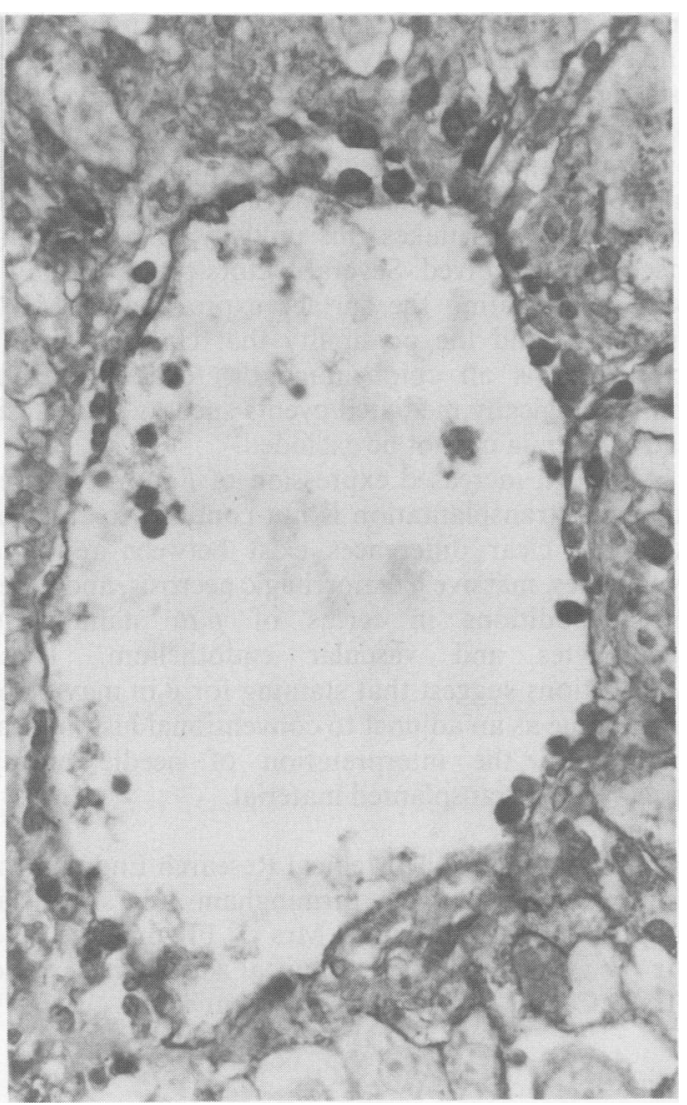

Fig 7 Endothelial $\beta_{2} m$ expression: (day 7 biopsy specimen). In this case of acute rejection hepatic venular endothelium shows strongly positive staining for $\beta_{2} m$. There is infiltration by lymphoid cells which also stain positively for $\beta_{2} m$.

were observed when cases of rejection (acute and chronic) and of massive haemorrhagic necrosis were compared with non-rejection biopsy specimens as a whole.

\section{Discussion}

The pattern of $\beta_{2} \mathrm{~m}$ expression in the "time-zero" biopsy specimens from the present study is similar to that previously described for MHC class $\mathrm{I}$ antigen expression in normal human liver. ${ }^{16}$ Staining was present most strongly in sinusoidal lining cells, more weakly in bile ducts and vascular endothelium, and was generally absent in hepatocytes. Focal membranous staining of hepatocytes was present in $30 \%$ of our baseline biopsy specimens and correlated with the presence and severity of cholestasis and hepatocyte necrosis in these specimens. While some previous studies failed to show the presence of class I antigens on normal hepatocyte membranes, ${ }^{16} 17$ others have shown weak staining in normal livers. ${ }^{78}$ Increased expression of $\beta_{2} \mathrm{~m}$ has also been described in association with intra- and extra-hepatic cholestasis ${ }^{719}$ and hepatocyte necrosis, ${ }^{8}$ these observations having been made both in transplanted livers and non-transplant biopsy specimens.

After liver transplantation we found a noticeably increased expression of $\beta_{2} \mathrm{~m}$ in bile ducts, hepatocytes and endothelial cells, with changes being present in biopsy specimens from all of the diagnostic categories examined. Only minor and mostly insignificant changes were observed in the staining of sinusoidal lining cells.

Increased expression of $\beta_{2} \mathrm{~m}$ in bile ducts occurred in cases of acute and chronic rejection, both of which are associated with histological evidence of bile duct damage. ${ }^{1-6}$ Increased display of class I antigens on cells renders them more susceptible to cytotoxic $T$ cell damage and is an attractive hypothesis to explain the bile duct damage that occurs in acute and chronic rejection. Bile duct expression often persisted in cases of resolving acute rejection, however, and was also a prominent finding in other post-transplant syndromes, particularly biliary obstruction. There was no significant difference in the degree of bile duct $\beta_{2} \mathrm{~m}$ staining among cases of rejection, resolving rejection, and non-rejection syndromes.

Expression of $\beta_{2} \mathrm{~m}$ on hepatocyte membranes was first reported as a feature of liver allograft rejection by Nagafuchi et al. ${ }^{7}$ Increased MHC class I antigen expression on hepatocytes has also been observed in acute rejection by So et al, ${ }^{8}$ who claimed this to be a specific feature of rejection. In contrast, Gouw et al found no significant difference in class I antigen expression on hepatocytes when comparing cases of rejection with other graft pathologies. We found an increased expression of $\beta_{2} \mathrm{~m}$ on hepatocytes in cases of acute rejection which partially resolved after successful immunosuppression treatment but which failed to return to baseline concentrations. Increased hepatocyte staining was also seen in several other graft states including chronic rejection. Overall, hepatocyte $\beta_{2} \mathrm{~m}$ staining was significantly higher in cases of rejection (acute and chronic) than in non-rejection syndromes. Interestingly, $\beta_{2} \mathrm{~m}$ expression in hepatocytes was often associated with cholestasismembranous staining and bile plugs being present in the same hepatocytes. Increased class I antigen expression on hepatocytes has been previously described in various non-transplant cholestatic syndromes including large duct obstruction, ${ }^{7}$ drug induced cholestasis, ${ }^{19}$ and primary biliary cirrhosis. ${ }^{19}$ Cholestasis is a prominent feature of many post-transplant syndromes including acute and chronic rejection, and membranous hepatocyte staining may therefore be an epiphenomenon related to cholestasis rather than a 
specific feature of rejection. The observation that bile plugs often stain positively for $\beta_{2} \mathrm{~m}$ and the correlation between cholestasis and membranous hepatocyte $\beta_{2} \mathrm{~m}$ display shown in "time zero" biopsy specimens might further support this hypothesis.

Previous studies of MHC expression after liver transplantation have concentrated mainly on hepatocytes, bile 'ducts, and sinusoidal lining cells, ${ }^{7-9}$ and have not specifically examined vascular endothelium. It is therefore interesting to note that our study showed vascular endothelium (particularly of portal and hepatic venules) to be the site in which the degree of $\beta_{2}$ m expression correlated most closely with histological features of rejection. This is not surprising in cases of acute rejection where inflammation of venular endothelium is a well recognised phenomenon. ${ }^{245}$ Venous endothelial inflammation has not been a prominent feature in our experience of acute rejection, ${ }^{6}$ but the relatively few lymphoid cells seen often displayed strong cytoplasmic and surface staining for $\beta_{2} \mathrm{~m}$. The finding of strong endothelial staining in cases of chronic rejection could also reflect continued endothelial damage. Histologically this is seen mainly in arteries which show a characteristic subendothelial foam cell proliferation ${ }^{113}$ rather than in veins which are histologically normal at this stage.

An interesting pattern of $\beta_{2} \mathrm{~m}$ expression was observed in six cases of massive haemorrhagic necrosis. We have recently described this as a specific syndrome of fulminant graft failure with distinctive clinical and histological features. ${ }^{10}$ The mechanisms underlying this condition are uncertain but several aetiologies including ischaemia, ${ }^{20}$ infection, ${ }^{21}$ rejection, ${ }^{22}$ and a Schwartzmann mechanism ${ }^{23}$ have been suggested. Five of our six cases showed extensive membranous hepatocyte staining, and in two there was a panacinar distribution which was not seen in any other post-transplant biopsy specimens. These two cases also showed extensive deposits of immunoglobulins in hepatocytes and sinusoids (unpublished observations). The clinical importance of these observations is uncertain but they could reflect a state of accelerated humoral mediated graft damage. A similar picture of massive haemorrhage and hepatocyte necrosis with only mild cellular infiltration has recently been described in an animal model of hyperacute humoral mediated rejection. ${ }^{24}$ Increased expression of $\beta_{2} \mathrm{~m}$ was also present in bile ducts and endothelial cells in our cases of massive haemorrhagic necrosis. Whether this represents evidence of immune mediated damage is uncertain, but these changes correlate with our histological findings which show endothelial inflammation and bile duct damage in some cases of massive haemorrhagic necrosis (unpublished observations).

The functional importance of increased $\beta_{2}$ m expression in the liver after transplantation is uncertain.
Increased MHC class I antigen expression in bile ducts and endothelial cells may have a role in the pathogenesis of rejection as these structures are the main targets of immune mediated damage. The considerable overlap that exists between rejection and other posttransplant syndromes in terms of $\beta_{2} \mathrm{~m}$ display in bile ducts, however, makes this unlikely to be the only mechanism involved. Several factors probably play a part in regulating the surface expression of MHC antigens, ${ }^{25}$ and the possibility that changed class I expression is an epiphenomenon related to nonimmunologically mediated events such as cholestasis and ischaemia cannot be excluded.

Although increased expression of $\beta_{2} \mathrm{~m}$ in the liver following transplantation is not confined to cases of rejection, clear differences exist between rejection syndromes, massive haemorrhagic necrosis, and other graft conditions in terms of $\beta_{2} \mathrm{~m}$ staining in hepatocytes and vascular endothelium. These observations suggest that staining for $\beta_{2} \mathrm{~m}$ may be of some value as an adjunct to conventional histological analysis in the interpretation of needle biopsy specimens of transplanted material.

We are grateful to the Medical Research Endowment Fund, University of Birmingham for financial support. We also thank Mrs J Fitzmaurice and $\mathrm{Mr} \mathrm{J}$ Gregory for technical assistance, and Mrs D Campbell for typing the manuscript.

\section{References}

1 Wight DGD. Pathology of rejection. In: Calne RY, ed. Liver transplantation: the Cambridge/Kings College experience. London: Grune and Stratton, 1983:247-77.

2 Snover DC, Sibley RK, Freese DK, et al. Orthotopic liver transplantation; a pathological study of 63 serial liver biopsies from 17 patients with special reference to the diagnostic features and natural history of rejection. Hepatology 1984;4:1212-22.

3 Vierling JM, Fennell RH. Histopathology of early and late human hepatic allograft rejection: evidence of progressive destruction of interlobular bile ducts. Hepatology 1985;5:1076-82.

4 Williams JW, Peters TG, Vera SR, Britt LG, Van Voorst SJ, Haggitt RC. Biopsy directed immunosuppression following hepatic transplantation in man. Transplantation 1985;39: 589-96.

5 Demetris AJ, Lasky S, Van Thiel DH, Starzl TE, Dekker A. Pathology of hepatic transplantation: a review of 62 adult allograft recipients immunosuppressed with a cyclosporin/ steroid regimen. Am J Pathol 1985;118:151-61.

6 Hubscher SG, Clements D, Elias E, McMaster P. Biopsy findings in cases of rejection of liver allograft. J Clin Pathol 1985;38:1366-73.

7 Nagafuchi Y, Hobbs KEF, Thomas HC, Scheuer PJ. Expression of beta-2-microglobulin on hepatocytes after liver transplantation. Lancet 1985; ;:551-4.

8 So SKS, Platt JL, Ascher NL, Snover DC. Increased expression of Class I major histocompatibility antigens on hepatocytes in rejecting human liver allografts. Transplantation 1987;43:79-85.

9 Gouw ASH, Houthoff HJ, Huitema S, Beelen JM, Gips CH, Poppema S. Expression of major histocompatibility complex antigens and replacement of donor cells by recipient ones in human liver grafts. Transplantation 1987;43:291-6. 
10 Hubscher SG. A proposed system for classifying rejectionassociated changes in serial liver biopsies obtained following liver transplantation. J Pathol 1987;152:225A.

11 Schardijn GHC, Van Eps LWS. B2-microglobulin: Its significance in the evaluation of renal function. Kidney Int 1987;32:635-41.

12 Maloy WL, Coligan JE. Is B2-microglobulin required for MHC Class I heavy chain expression. Immunology Today 1985;6: 263-4.

13 Grond J, Gouw ASH, Poppema S, Slooff MJH, Gips GH. Chronic rejection in liver transplants: A histopathologic analysis of failed grafts and antecedent serial biopsies. Transplant Proc 1986;18(Suppl 4):128-35.

14 Poulsen H, Christofferson P. Atlas of liver biopsies. Munksgaard: JB Lippincott Company, 1979:100-1.

15 Hubscher SG, Adams DH, Buckels JAC, McMaster P, Neuberger J, Elias E. Massive haemorrhagic necrosis of the liver following liver transplantation. Gut 1987;28:A1344-5.

16 Barbatis C, Woods J, Morton JA, Fleming KA, McMichael A, McGee J O'D. Immunohistochemical analysis of $\operatorname{HLA}(A, B, C)$ antigens in liver disease using a monoclonal antibody. Gut 1981;22:985-91.

17 Lautenschlager I, Hayry P. Expression of the major histocompatibility complex antigens on different liver cellular components. Scand J Immunol 1981;14:421-6.

18 Daar AS, Fuggle SV, Fabre JW, Ting A, Morris PJ. The detailed distribution of HLA-A,B,C antigens in normal human organs. Transplantation 1984;38:287-92.
19 Nagafuchi Y, Scheuer PJ. Hepatic B2-microglobulin distribution in primary biliary cirrhosis. $J$ Hepatol 1986;2:73-80.

20 Starzl TE, Groth CG, Brettschneider L, et al. Orthotopic homotransplantation of the human liver. Ann Surg 1968;168:392-415.

21 Starzl TE, Iwatsuki S, Van Thiel DH, et al. Evolution of liver transplantation. Hepatology 1982;2:614-36.

22 Starzl TE, Putnam CS. Experience in hepatic transplantation. Philadelphia: WB Saunders Co, 1969:283-94.

23 Portmann B, O'Grady J, Williams R. Disease recurrence following orthotopic liver transplantation. Transplant Proc 1986;18(Suppl 4):136-42.

24 Knechtle SJ, Kolbeck PC, Tsuchimoto S, Coundouriotis A, Sanfilippo F, Bollinger RR. Hepatic transplantation into sensitized recipients. Demonstration of hyperacute rejection. Transplantation 1987;43:8-12.

25 Pober JS, Collins T, Gimbrone MA, Libby P, Reiss CS. Inducible expression of class II major histocompatibility complex antigens and the immunogenicity of vascular endothelium. Transplantation 1986;41:141-6.

Requests for reprints to: Dr S G Hubscher, Department of Pathology, The Medical School, University of Birmingham, Birmingham B15 2TJ, England. 